OPEN ACCESS

Edited by:

Wiebke Kallenborn-Gerhardt, Goethe-University Frankfurt, Germany

Reviewed by: Zhouguang Wang, Albert Einstein College of Medicine, United States

Aruna Sharma,

Uppsala University, Sweden

*Correspondence: Sheng Wang wangthomas2002@163.com Huazi Xu

spinexu@163.com

Xiaolei Zhang

zhangxiaolei@wmu.edu.cn

${ }^{t}$ These authors have contributed equally to this work

Specialty section: This article was submitted to Inflammation Pharmacology,

a section of the journal

Frontiers in Pharmacology

Received: 18 September 2019 Accepted: 07 May 2020 Published: 04 June 2020

Citation:

Zheng G, Zheng F, Luo Z, Ma H, Zheng $D$, Xiang $G, X u C$, Zhou Y, WuY, Tian N, Wu Y, Zhang T, Ni W, Wang S, $X u H$ and Zhang $X$ (2020) CO-Releasing Molecule (CORM)-3

Ameliorates Spinal Cord-Blood Barrier Disruption Following Injury to the Spinal Cord.

Front. Pharmacol. 11:761. doi: 10.3389/fphar.2020.00761

\section{CO-Releasing Molecule (CORM)-3 Ameliorates Spinal Cord-Blood Barrier Disruption Following Injury to the Spinal Cord}

\author{
Gang Zheng ${ }^{1,2 \dagger}$, Fanghong Zheng ${ }^{1,2,3 \dagger}$, Zucheng Luo ${ }^{1,2 \dagger}$, Haiwei Ma ${ }^{1,2}$, \\ Dongdong Zheng ${ }^{4}$, Guangheng Xiang ${ }^{1,2}$, Cong Xu ${ }^{1,2}$, Yifei Zhou ${ }^{1,2,3}$, Yaosen $W^{1,2,3}$, \\ Naifeng Tian ${ }^{1,2,3}$, Yan $\mathrm{Wu}^{5}$, Tan Zhang ${ }^{6}$, Wenfei $\mathrm{Ni}^{1,2}$, Sheng Wang ${ }^{1,2,3^{*}}$, Huazi $\mathrm{Xu}^{1,2,3^{*}}$ \\ and Xiaolei Zhang ${ }^{1,2,3,7 *}$

\begin{abstract}
${ }^{1}$ Department of Orthopaedics, The Second Affiliated Hospital and Yuying Children's Hospital of Wenzhou Medical University, Wenzhou Medical University, Wenzhou, China, ${ }^{4}$ Department of Neurosurgery, The Second Affiliated Hospital and Yuying Hospital, Shaoxing, Wenzhou, China, ${ }^{7}$ Department of Orthopaedics, Chinese Orthopaedic Regenerative Medicine Society, Hangzhou, China
\end{abstract} \\ Wenzhou, China, 2 Zhejiang Provincial Key Laboratory of Orthpaedics, Wenzhou, China, ${ }^{3}$ The Second School of Medicine, \\ Children's Hospital of Wenzhou Medical University, Wenzhou, China, ${ }^{5}$ Department of Orthopaedics, The Second Affiliated \\ Hospital, School of Medicine, Zhejiang University, Wenzhou, China, ${ }^{6}$ Department of Orthopaedics, Shaoxing People's
}

Spinal cord injury $(\mathrm{SCl})$ is a clinical tough neurological problem without efficient cure currently. Blood-spinal cord barrier (BSCB) interruption is not only a crucial pathological feature for $\mathrm{SCl}$ process but is a possible target for future SCl treatments; however, few treatments have been developed to intervene BSCB. In the present study, we intravenously injected CO-releasing molecule3 (CORM-3), a classical exogenous CO donor, to the rats experiencing $\mathrm{SCl}$ and assessed its protection on BSCB integrity in rats. Our results demonstrated that the exogenous increasing of $\mathrm{CO}$ by CORM-3 blocked the tight junction (TJ) protein degeneration and neutrophils infiltration, subsequently suppressed the BSCB damage and improved the motor recovery after SCl. And we certified that the CO-induced down-regulation of MMP-9 expression and activity in neutrophil might be associated with the NF-кB signaling. Taken together, our study indicates that CO-releasing molecule (CORM)-3 ameliorates BSCB after spinal cord injury.

Keywords: spinal cord injury, blood spinal cord barrier, carbon monoxide, neutrophil, co-releasing molecule-3

\section{INTRODUCTION}

Spinal cord injury (SCI) causes longstanding physical and sensory impairment and can be fatal. Traumatic SCI consists of two stages: (1) the primary injury refers to the immediate local mechanical impact and (2) the inferior injury is identified as a multifaceted cascade representing for local edema, hemorrhage, oxidative stress, inflammation, and apoptosis (Penas et al., 2010). Alleviating and delaying the secondary injury process is identified as the potential target for SCI treatment (Giszter, 2008). But previous studies principally focus on neuronal death and 


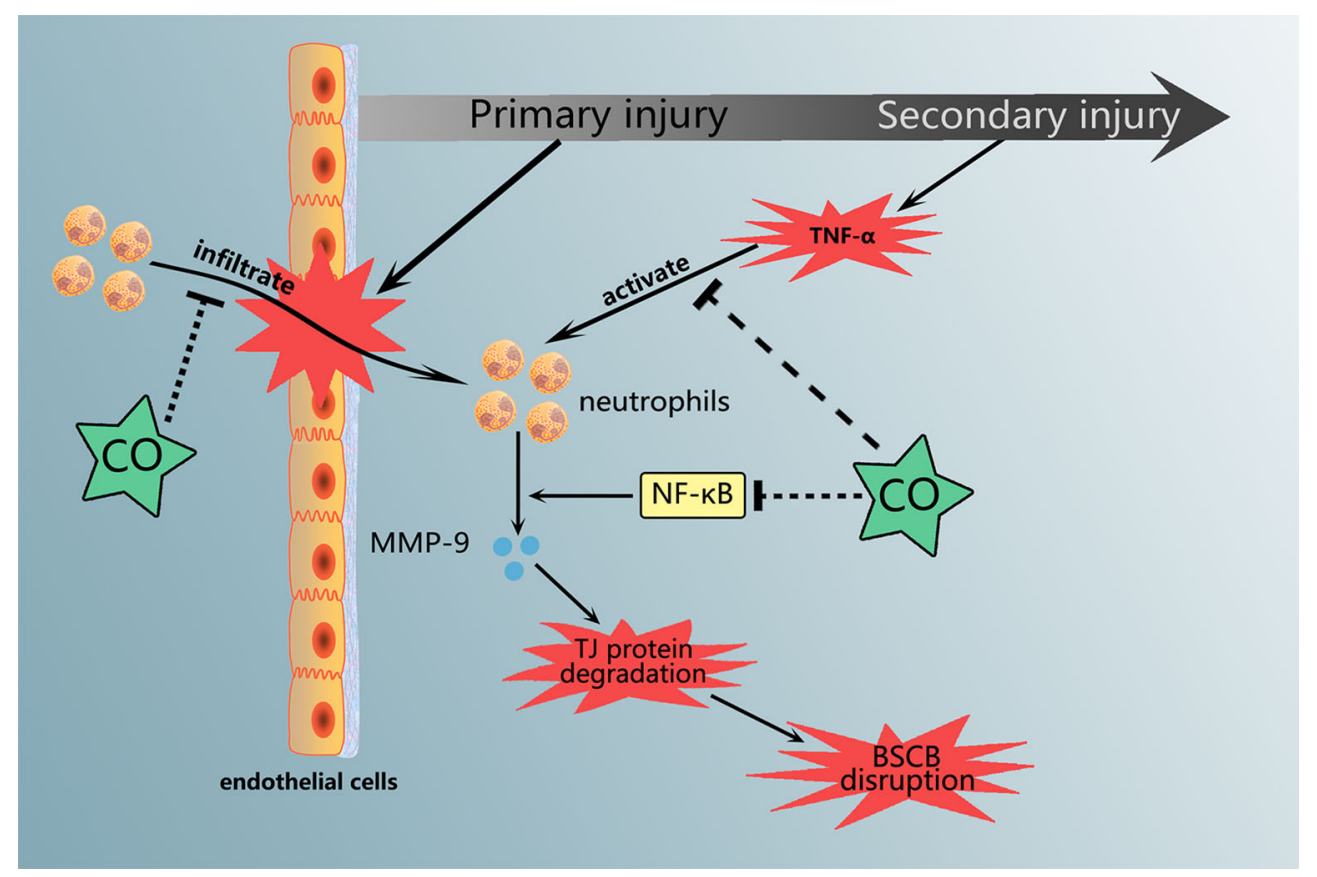

GRAPHICAL ABSTRACT | Carbon monoxide treatment attenuated the neutrophils infiltration and MMP-9 expression and activation, which are associated with NF$\kappa \mathrm{B}$ pathway.

regeneration. The effect of blood spinal cord barrier (BSCB) for this process has yet to be sufficiently investigated (Fassbender et al., 2011; Sharma, 2011).

Under normal physiological condition, the BSCB could prevent the entry of pathogens, blood-derived products, and cells into the spinal cord to maintain homeostasis within the spinal cord (Bartanusz et al., 2011; Kumar et al., 2016). The barrier function bases on non-honeycomb shaped endothelial cells (ECs) and their additional constituents such as pericytes and astrocytic end feet (Palmer, 2010). ECs in the central nervous system (CNS) characterize by plentiful cytosolic mitochondria and the absence of cell membrane fenestrations and pinocytic vacuoles (Bartanusz et al., 2011). Meanwhile, ECs produce tight junction (TJ) proteins to form the complicated network, which restricts the paracellular diffusion pathway between the ECs. These structural features ensure the formation of stable capillary microenvironment (Obermeier et al., 2013). Unfortunately, primary injury causes irreversibly local BSCB disruption, which allows the inflammatory cells (primarily neutrophils) infiltrate and migrate into the uninjured section and subsequently exacerbates the secondary sequential damage by secreting neurotoxic factors such as various matrix metallopeptidases (MMPs), reactive oxygen species (ROS), and inflammatory cytokines (Sharma, 2011; Kumar et al., 2016). The abnormal expression and activation of MMPs, especially MMP-9 could promote the $\mathrm{TJ}$ proteins degeneration, the fundamental molecular components of BSCB (Yu et al., 2008; Lee et al., 2012). The genetic or pharmacological suppression of MMP expression and activation or direct blocking the neutrophil infiltration have been demonstrated the protection of BSCB following SCI (Noble et al., 2002; Wells et al., 2003; Yu et al., 2008; Kumar et al., 2016).

Heme oxygenase $(\mathrm{HO})$ catalyzes heme degradation with the $\mathrm{CO}$ production, which has been proved with biological function including anti-inflammation, anti-apoptosis, and anti-oxidative at low dose (Kim et al., 2007; Motterlini and Otterbein, 2010; Wang et al., 2010). In 2009, Zeynalov and Dore found CO inhalation reduced infarct volume and attenuated brain edema following mouse ischemic brain injury (Zeynalov and Doré, 2009). Most recently, Choi et al. reported that the COreleasing molecule (CORM)-3 treatment, the exogenous $\mathrm{CO}$ donor, ameliorated the prognosis of traumatic brain injury (TBI) by promoting neurogenesis through the rescuing the crosstalk between the pericyte and neural stem cell (Choi et al., 2016). Nevertheless, the studies about CO effect in SCI mainly focus on the HO. Applying hemin, chemicals, and natural products to regulate $\mathrm{HO}$ expression or activity subsequently inducing the $\mathrm{CO}$ production, which not have enough evidence to confirm the CO effect for BSCB (Lin et al., 2016; Lin et al., 2017; Lu et al., 2018; Wei et al., 2018; Zheng et al., 2019). Therefore, we performed this research to make up for this question.

Recently, our group reported that increasing CO by CORM-3 alleviated neurological deficits and improved the functional recovery after SCI. And we detected $\mathrm{CO}$ level and $\mathrm{HO}-1$ expression variation within 7 days following SCI (Zheng et al., 
2019). In this article, we assessed the protection of CO in BSCB destruction. And CORM-3 treatment attenuated the neutrophils infiltration and MMP-9 expression and activation in vivo and in vitro. And we initially examined the potential mechanism for CO-regulated MMP-9 production in neutrophil, which might be associated with NF- $\mathrm{\kappa B}$ pathway.

\section{METHODOLOGY AND MATERIALS}

\section{Antibodies and Chemical Reagents}

Carbon monoxide releasing molecule 3 (CORM-3) were obtained from Sigma-Aldrich (St. Louis, MO, USA). Antibody against ZO-1 and Occludin were bought at Santa Cruz Biotechnology (Santa Cruz, CA, USA). Antibody against Ly6G, p-p65, p65, GAPDH, MMP-2/-3/-9, and Lamin B were bought at Abcam (Cambridge, CA, USA). TNF- $\alpha$ and anti-ICAM- 1 were purchased from R\&D system (Minneapolis, MN, USA). AntiMPO was bought at Bioworld (Minneapolis, MN, USA)

\section{The SCl and Animal Model}

The Sprague-Dawley female adult rats (220-250 g, 2 months old) were bought at the Animal Center of the Chinese Academy of Sciences in Shanghai, China. The ethical procedures for handling the rats referred to the guidelines and permission from the Animal Care and Use Committee of Wenzhou Medical University (ethic code: wydw2014-0129).

The rats were randomized into four groups and they were given intraperitoneal sodium pentobarbital injections $(65 \mathrm{mg} / \mathrm{kg})$ for sedation. After their skins were prepared and sterilized at the back, skin incisions were done lengthwise on the dorsal midline to reveal the vertebral column and they underwent surgical removal of the lamina at the T9 level. The visible spinal cord underwent a simulated crush injury by compressing it with a vascular clasp (30 g force; Oscar, China) for a minute. Surgery consisting of a laminectomy at level T9 without exposure to spinal crush injury, was also performed in the rats of Sham group. The post-surgical care consisted of manually emptying the urinary bladder two times a day the normal bladder function returned and treatments consisting of cefazolin sodium (50 mg/ $\mathrm{kg}$ ). The rats that experienced SCI were randomized into three groups; group treatments included CORM-3/iCORM-3/saline. To investigate the effects of CO, preparations of inactive CORM3 (iCORM-3) were made by placing CORM-3 in saline $(\mathrm{pH}=$ 7.4) overnight at room temperature to complete the $\mathrm{CO}$ releasing process (Zhang W. et al., 2017). The CORM-3 underwent dilutions involving normal saline and completed an ultimate CORM-3 concentration of $8 \mathrm{mg} / \mathrm{ml}$. The CORM-3 solution was instantly injected to the rat tail veins after surgery with a dosage of $8 \mathrm{mg} / \mathrm{kg}$ per day until they were euthanized. Corresponding quantities of iCORM-3 and normal saline were injected for vehicle regulation.

\section{Examination Using Evans Blue Dye}

The BSCB permeability was detected by Evans blue dye as previously described (Zhang D. et al., 2017). Three days after injury to spinal cord, the rats underwent injections of $2 \%$ Evans blue dye $(2 \mathrm{ml} / \mathrm{kg}$ ) mixed in saline solution intravenously via the veins of the tail section. After $2 \mathrm{~h}$, rats were euthanized with sodium pentobarbital (65 mg/kg, i.p.), then underwent perfusion in $0.9 \%$ normal saline. The lesioned spinal cord EB tissues were quantified and absorbed in $\mathrm{N}, \mathrm{N}^{\prime}$-dimethylformamide (Jinsan, Wenzhou, China) at $50^{\circ} \mathrm{C}$ for $72 \mathrm{~h}$. The supernatant's optical density was investigated using an enzyme-labeled meter (at an excitation wavelength of $620 \mathrm{~nm}$ and an emission wavelength of $680 \mathrm{~nm}$ ). The presence of the dye in samples indicated existence of $\mathrm{lg} / \mathrm{g}$. After being injected with EB, the rats were subjected to fixation via perfusion with $4 \%$ paraformaldehyde for $2 \mathrm{~h}$. The frozen section machine cut the spinal cord tissues into segments of $10 \mu \mathrm{m}$ thickness at $20^{\circ} \mathrm{C}$, then the segments underwent observation using a fluorescent microscope (Olympus, Tokyo, Japan).

\section{Gelatin Zymography}

Gelatin zymography was done as mentioned earlier. The MMP2,9 activity in vivo at day 3 after SCI was detected by Gelatin Zymography. The core of lesioned spinal cord ( $0.5 \mathrm{~cm}$ in length) was standardized in a lysis buffer consisting of $50 \mathrm{mM}$ Tris- $\mathrm{HCl}$, pH 8.0, $150 \mathrm{mM} \mathrm{NaCl}, 1 \% \mathrm{NP}-40,0.5 \%$ deoxycholate, and $0.1 \%$ SDS. Equal protein quantities (40 ug) were put on $10 \%$ SDSpolyacrylamide gel electrophoresis, co-polymerized with $1 \mathrm{mg} /$ $\mathrm{ml}$ gelatin (Sigma-Aldrich). The gel was subjected to washing in 2.5\% Triton X-100 after electrophoresis for $30 \mathrm{~min}$ and underwent incubation for $24 \mathrm{~h}$ at $37^{\circ} \mathrm{C}$ in substrate buffer, together with Tris $50 \mathrm{mM} / \mathrm{l}(\mathrm{pH} 7.6), \mathrm{CaCl} 25 \mathrm{mM}, \mathrm{NaCl} 0$. $2 \mathrm{mM}$, and $0.02 \%(\mathrm{w} / \mathrm{v})$ Brij-35 (Sigma-Aldrich). Finally, the Coomassie blue solution stained the gel for $4 \mathrm{~h}$ and underwent de-staining with $10 \%$ acetic acid/40\% methanol. The gels underwent scanning for quantitative examination and the positive band was quantified by NIH Image J software (NIH). In vitro, MMP-9 in regulated media was investigated using gelatin zymography as mentioned earlier.

\section{Human Brain Microvascular Endothelial Cells Culturing and Treatment}

Human brain microvascular endothelial cells (HBMECs) and endothelial cell medium were bought at ScienCell Research Laboratories (ScienCell Research Laboratories, San Diego, CA, USA). Cells were incubated in a moistened environment consisting of $5 \% \mathrm{CO} 2$ and $95 \%$ air at $37^{\circ} \mathrm{C}$. Cells were pretreated for $6 \mathrm{~h}$ with CORM-3 $(100 \mu \mathrm{m})$.

\section{Primary Neutrophil Culturing and Treatment}

The Percoll gradient was laminated with SD adult rat's (200-250 g) blood in layer densities of 45,54 , and $63 \%$. To detect the neutrophils between the second and third layer, the blood was prepared using gradient centrifuge (3,000 rpm for $15 \mathrm{~min}$ ). The erythrocyte lysis and ACK buffer purified the cells. The granulocytes underwent washing in HBSS buffer and diluting in RPMI1640 media with 10\% $(\mathrm{v} / \mathrm{v})$ heat-inactivated FBS followed by plating the neutrophils in the six-well dish with a density of 100,000 cells $/ \mathrm{cm}^{2}$ (Pashevin et al., 
2015). The TNF- $\alpha(100 \mathrm{ng} / \mathrm{ml})$ and CORM-3 $(100 \mu \mathrm{m})$ were used to treat the neutrophils for $6 \mathrm{~h}$.

\section{Co-Culture of HBMECs and Neutrophils}

One part of HBMECs were pretreat with CORM-3 $(100 \mu \mathrm{m})$ for $6 \mathrm{~h}$ and then washed out followed by addition of neutrophils to the HBMECs cultures at a concentration of $1 \times 10^{6}$ cells per $\mathrm{ml}$. In addition, one part of these neutrophils had been pretreated with TNF-a $(100 \mathrm{ng} / \mathrm{ml})$ for $6 \mathrm{~h}$. The unstimulated as well as the activated neutrophils were harvested, washed, and then added to the HBMECs cultures as concentrate. The HBMEC-neutrophil co-culture were maintained at $37^{\circ} \mathrm{C}$ for $6 \mathrm{~h}$ and then neutrophils were washed out. Finally, HBMECs were collected and processed for western blotting analyses and immunofluorescence staining.

\section{Western Blot Assay}

RIPA lysis buffer consisting of $1 \mathrm{mM}$ PMSF extracted whole proteins then their concentrations were quantified by the Enhanced BCA Protein Assay Kit (both from Beyotime, Shanghai, China) via a Microplate Reader (Molecular Devices Flexstation 3, USA). A protein tissue of $40 \mathrm{ng}$ was parted by sodium dodecylsulfate-polyacrylamide gel electrophoresis (SDS PAGE) and transported to a polyvinylidene difluoride membrane (Bio-Rad, California, USA). The 5\% nonfat milk was used for blocking process for $2 \mathrm{~h}$, then the membranes underwent incubation with the primary antibodies against ZO-1 (1:500), Occludin (1:500), ICAM-1 (1:1,000), MPO (1:500), MMP-2/-3 and -9 (1:1,000 respectively), p65 (1:500), Lamin B $(1: 1,000)$, GAPDH $(1: 5,000)$. The membranes underwent washing with TBS thrice for $5 \mathrm{~min}$, followed by treatment in horseradish peroxidase-conjugated secondary antibodies. After washing thrice in TBST, the visualization of the blots was done via electrochemiluminescence plus reagent (Invitrogen, Carlsbad, USA). In the final phase, the Image Lab 3.0 software measured the blot intensities (Bio-Rad, California, USA).

\section{Immunofluorescent Staining}

Three days after injury to the spinal cord, the tissue samples were collected. After harvesting, the spinal cord segments were fixed in $4 \%$ PFA, followed by washing and then embedded in paraffin. The transverse segments of $5 \mu \mathrm{m}$ thickness underwent cutting, deparaffining in xylene, and rehydration in ethanol rinses. The segments underwent incubation in $10 \%$ normal goat serum for 1 $\mathrm{h}$ at room temperature in PBS consisting of $0.1 \%$ Triton X-100. This was followed by incubation using suitable primary antibodies overnight at $4^{\circ} \mathrm{C}$ in a similar buffer. Based on their varying targets, primary antibodies that were used included: Ly6G (1:100) and MMP-9 (1:100). After incubating the primary antibodies, segments underwent washing for $40 \mathrm{~min}$, followed by incubation with Alexa Fluor 488/594 goat antirabbit/mouse secondary antibodies at room temperature for $1 \mathrm{~h}$. The sections underwent rinsing thrice with PBS and incubation with 4,6-diamidino-2-phenylindole (DAPI) for 10 min, followed by washing in PBS and a coverslip sealed them. The fluorescent microscope (Olympus Inc., Tokyo, Japan) recorded the images, then three investigators quantified the positive neurons in each segment while being blinded to the groups under investigation. The values from the counting of 3040 random sections produced the calculations for the rates of corresponding-protein positive cells per section examining five rats each group.

\section{Statistical Analysis}

The outcomes were represented by mean \pm S.D. The SPSS statistical software program 20.0 (IBM, Armonk, NY, USA) analyzed the data. The one-way analysis of variance (ANOVA) and Tukey's test were used to compare the experimental groups. The BBB scores underwent analysis using Mann-Whitney test. Statistical values of $P<0.05$ were stated as significant.

\section{RESULTS}

\section{Carbon Monoxide Attenuates BSCB Disruption and TJ Protein Loss Following $\mathrm{SCl}$}

Recently, we have demonstrated CORM-3 treatment alleviated neurological deficits and improved the functional recovery after SCI via increasing the CO content in spinal cord (Zheng et al., 2019). In this article, we mainly discussed CO protection in BSCB function after SCI.

Evan's Blue dye to test BSCB permeability via tail vein at 3 days following SCI. By the Evan's Blue staining, less blue dye appears around the damaged area in CORM-3 group relative to the SCI and iCORM-3 groups as well as the quality results in Figures 1A, B. Meanwhile, compared to the SCI and ICORM-3 groups, CORM-3 treatment weakened the red fluorescence intensity in the transverse and longitudinal section of spinal cord (Figures 1C, D). As the fundamental molecular structure of BSCB, SCI reduces ZO-1, and Occludin protein expression, whereas CORM-3 reversed this trend (Figures 1E, F).

\section{CO Reduces the MMP-9 Production and Blocks Neutrophil Infiltration After SCI}

During the SCI, the abnormal increase of MMPs production and activity contribute to TJ protein degradation (Lee et al., 2014; Kumar et al., 2017; Wang et al., 2017). By western blot assay, SCI-induced upregulation of MMP-2, -3, and -9 expression was significantly reduced by CORM-3 treatment (Figures 2A, B). And the gelatin zymography results revealed the CORM-3 obviously suppressed the activation of MMP-2 and -9 (Figures 2C, D).

As the primary source of MMP-9 in the early stage post-SCI, infiltrating neutrophil was measured by western blot and immunofluorescence (de Castro et al., 2000; Neirinckx et al., 2014). As shown in Figures 2E, F, the expression of intercellular adhesive molecule 1 (ICAM-1), linked to leukocyte adhesion, and Myeloperoxidase (MPO), a peroxidase enzyme primarily expressing in neutrophils and storing in azurophilic granules 


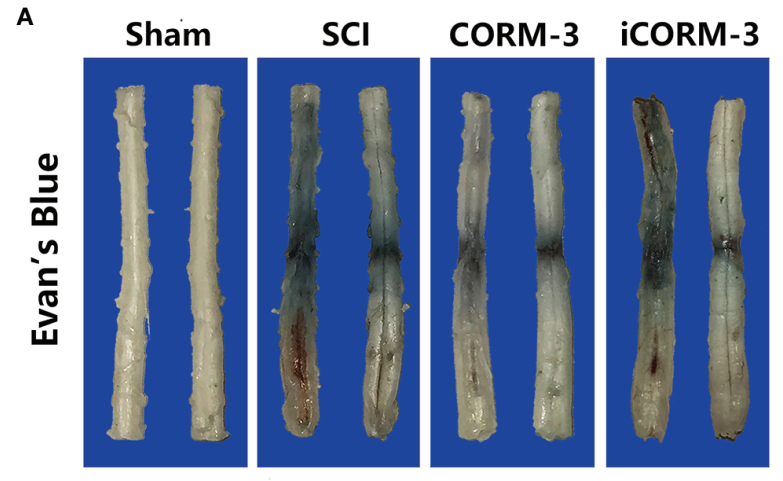

C

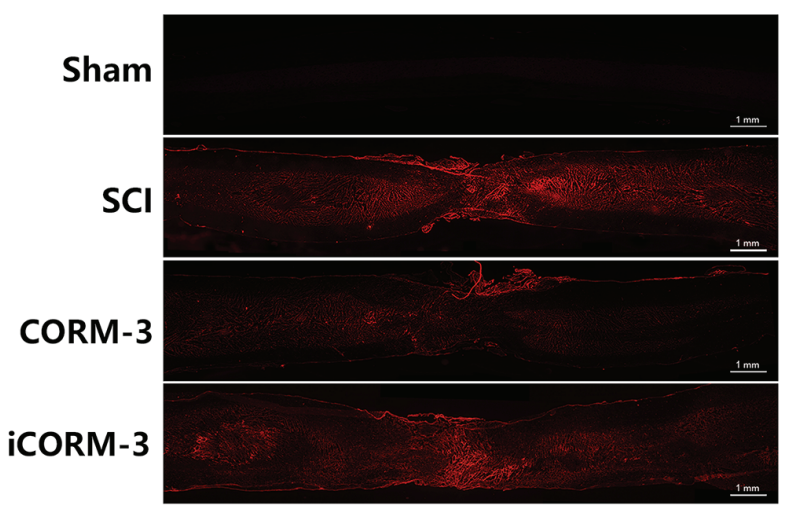

E

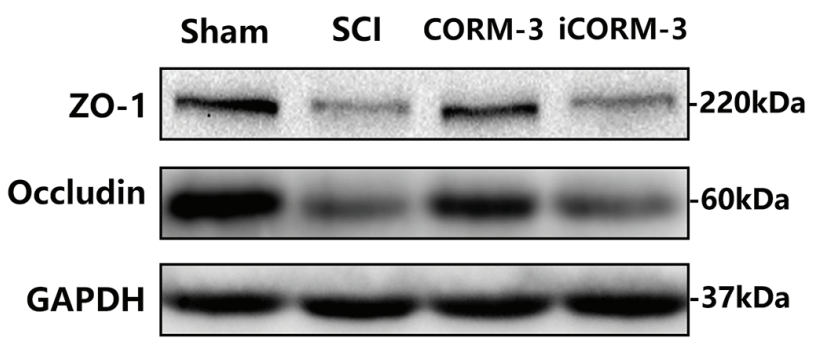

B

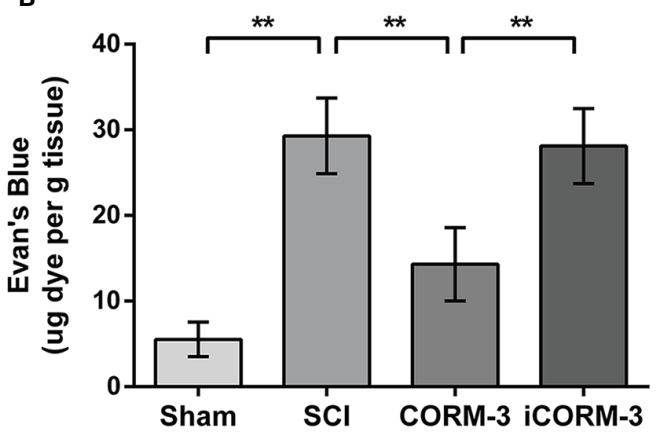

D

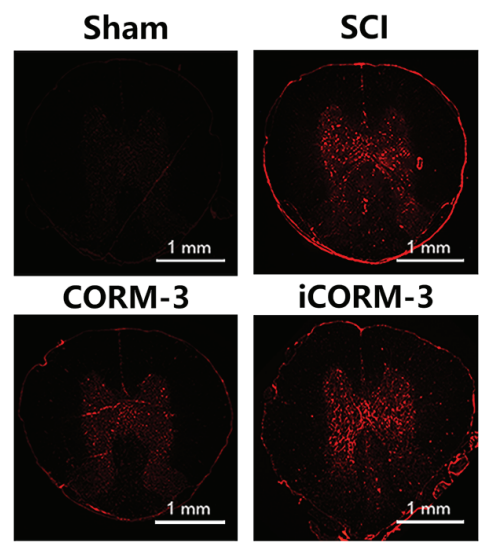

$\mathbf{F}$

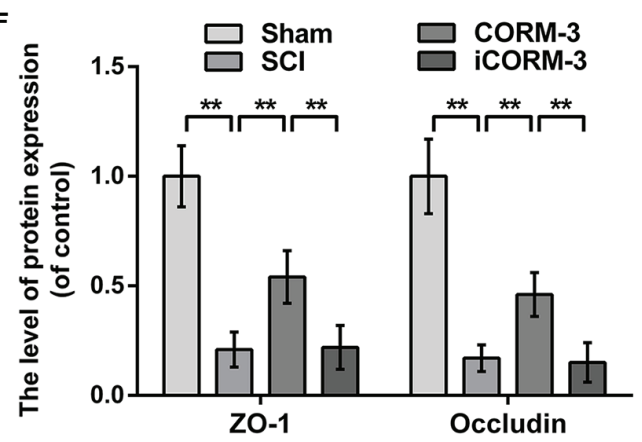

FIGURE 1 | Carbon monoxide inhibits BSCB interruption and declined TJ proteins at 3 days following SCl. (A) Characteristic complete spinal cords show that Evan's Blue dye infused into spinal cord at 3 days. (B) Quantifying the Evan's Blue at 3 days ( $\mu \mathrm{g} / \mathrm{g})$. (C, D) Characteristic fluorescent images of Evans Blue Dye extravasation at 3 days. (bars: $1 \mathrm{~mm}$ ). (E, F) The protein expression of ZO- 1 and Occludin in spinal cord at 3 days post-SCl. All data is denoted as mean \pm S.D. ( $\mathrm{n}=$ 5). ${ }^{*} P<0.01$

were increased after SCI, whereas CORM-3 treatment alleviated these phenomena (Neirinckx et al., 2014; Patterson et al., 2014). The outcomes of MPO activity assay were similar to the western blot (Figure 2G). To determine the infiltration levels of neutrophils following SCI, we performed a double-labeling immunofluorescence staining with MMP-9 and Ly6G (a neutrophil marker). As shown in Figures 2H, I, CORM-3 injection decreased the number of Ly6G-positive cells on 3 days post-SCI. Furthermore, we can obviously discovered that the MMP-9 and Ly6G fluorescent could basically be collocated, which again answers the source of the MMP-9.

\section{Regulation of MMP-9 Expression and Activation by $\mathrm{CO}$ in Neutrophils Is Related to In Vitro NF-KB Signaling Pathway}

To investigate the mechanism of CORM-3-medicated MMP-9 regulation, we extracted neutrophils from blood and stimulated with TNF- $\alpha$ to imitate the SCI model in vitro. The western blot, 
A

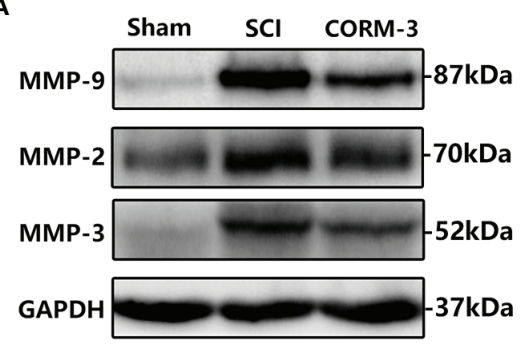

C

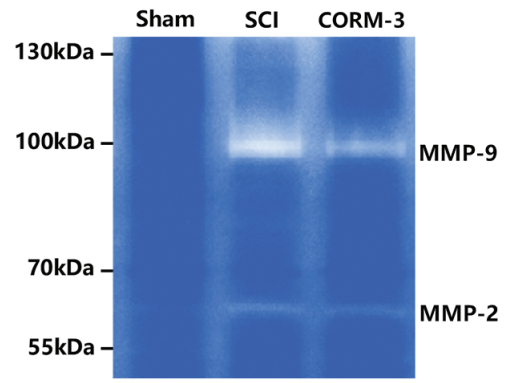

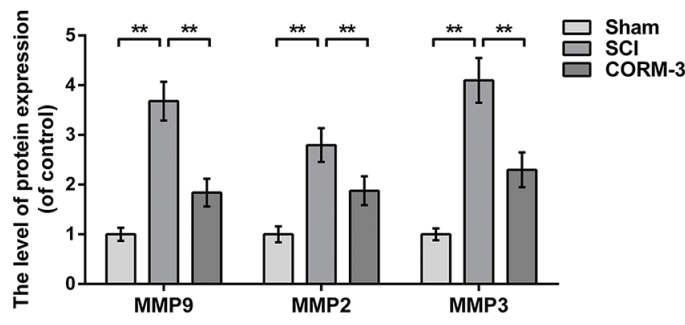

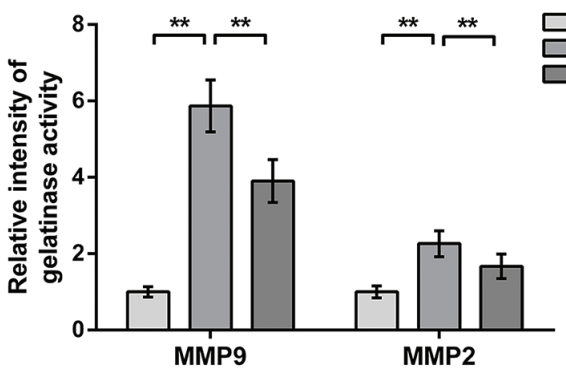

E

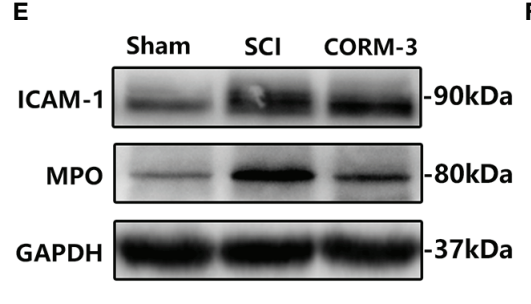

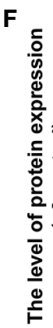

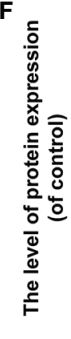

H
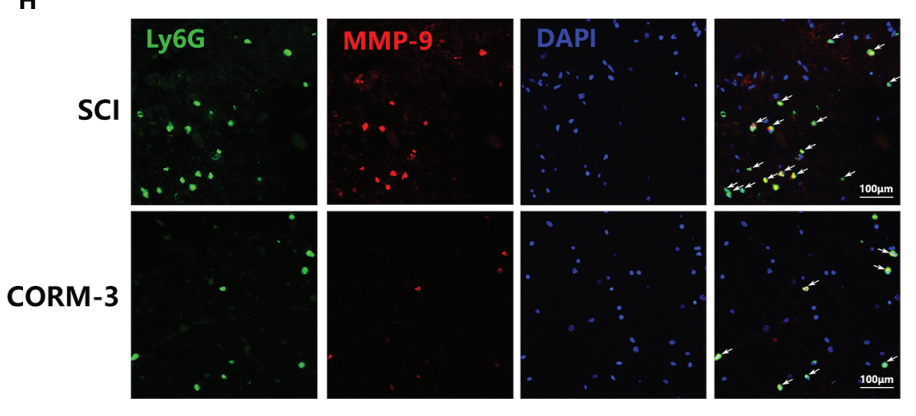

I

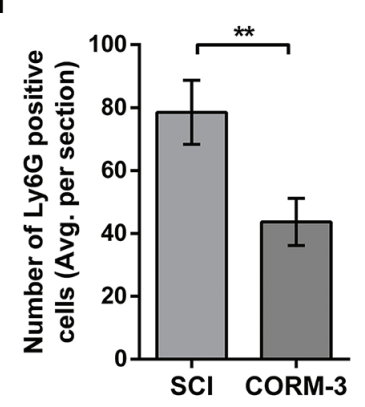

FIGURE 2 | CORM-3 blocks the neutrophil infiltration and inhibits MMP-9 production and activation at 3 days after SCI. (A, B) The protein expression of ICAM-1 and MPO in spinal cord at 3 days post-SCl. (C) MPO activity in spinal cord at 3 days post-SCl. (D, E) The protein expression of MMP-2, -3 , and -9 in spinal cord at 3 days post-SCl. (F, G) Dual immunofluorescence of MMP-9 and Ly6G in segments from tissues at 3 days post-SCl (bar: 50 um). The white arrow indicates the Ly6G/MMP-9 positive cells. (H, I) Representative zymography and measurement data of MMP-2 and -9 in spinal cord at 3 days post-SCl. All data is denoted as mean \pm S.D. $(n=5) .{ }^{\star *} P<0.01$.

gelatin zymography, and MMP-9 immunostaining outcomes illustrated that CORM-3 inhibited TNF- $\alpha$-stimulated MMP-9 expression and activate neutrophils, which are consistent with our in vivo results (Figures 3A-E).

In neutrophils, NF- $\mathrm{KB}$ signaling is essential in MMP-9 synthesis and activation (Tahanian et al., 2011). TNF- $\alpha$ causes the p65 transfer to the nucleus and next initiate transcription and translation of MMP-9 (Dilshara et al., 2015). We investigated the levels of p65 expression in the cytoplasmic and nuclear portions of neutrophils. After TNF- $\alpha$ stimulation, there were elevated nuclear p65 expressions then the cytoplasmic p65 were declined, whilst CORM-3 treatment inhibited this response (Figures 3F, G). These data indicated that NF- $\mathrm{\kappa B}$ pathway could be considered as the potential mechanism of CORM-3 protection. Additionally, our in vivo experiments also showed that CORM-3 treatment inhibited NF- $\kappa$ B pathway activation after SCI (Figure S1). 

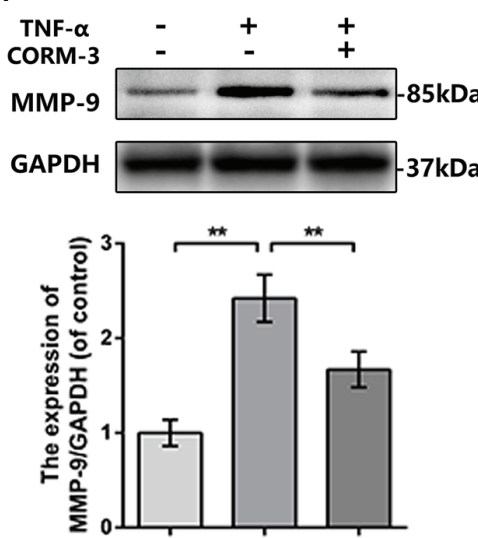

C
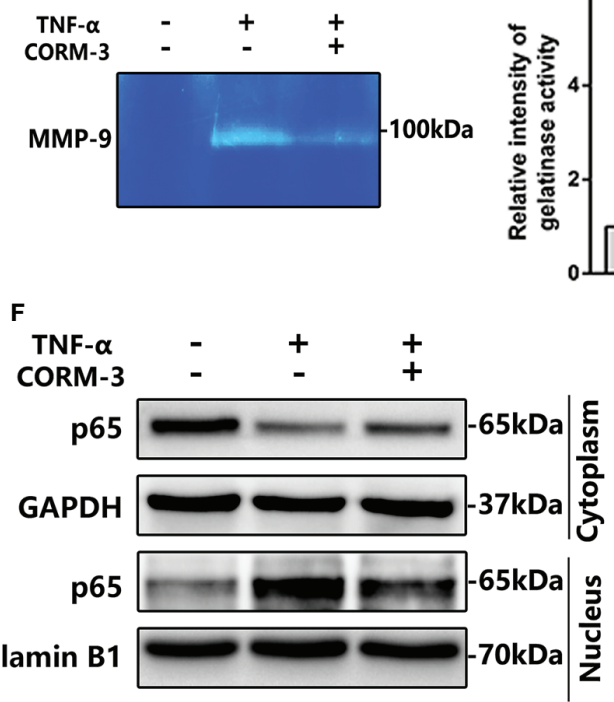
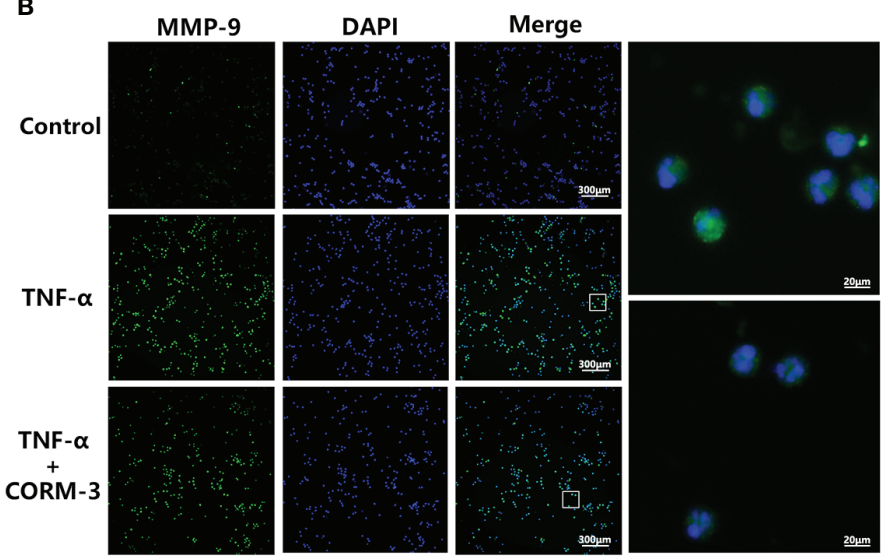

E
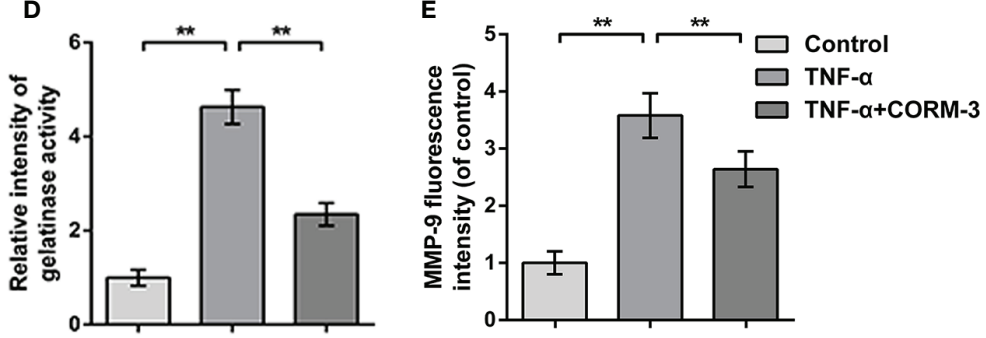

G

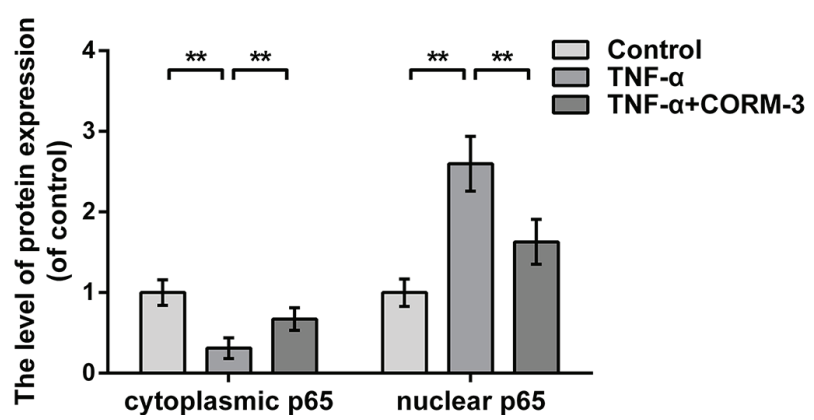

FIGURE 3 | MMP-9 regulation of CORM-3 is related to NF- $\mathrm{kB}$ signaling pathway in neutrophils in vitro. (A) The protein expression of MMP-9 in neurons were treated as above. (B) The immunofluorescence of MMP-9 in the neutrophils. (bars: $300 \mu \mathrm{m}$ or $20 \mu \mathrm{m})$. (C, D) Representative zymography and measurement data of MMP-2 and -9 in neutrophils treated as above. (E) Quantification of the fluorescence intensity of MMP-9 in neutrophils treated as above. (F, G) The protein expression of p65 in neutrophils treated as above. All data is denoted as mean \pm S.D. $(n=5)$. ${ }^{\star \star} P<0.01$.

CORM-3 Alleviates TJ Protein Degradation Induced by Activated Neutrophils in HMBECs In Vitro

To explain the CO-induced protection, we pretreated HBMECs by CORM-3 and then co-cultured with neutrophils. In our experimental settings (Figure 4A), we used TNF- $\alpha$ to induce neutrophils activation and removed after $6 \mathrm{~h}$ incubation. Neutrophils were harvested, washed, and added to HBMEC as concentrate. Before adding neutrophils, one part of HBMECs was pretreated with CORM-3. As shown in Figures 4B, C, activated neutrophils caused prominent loss of ZO-1 and Occludin in HBMECs, whereas CORM-3 pretreatment in HBMEC reversed it. Interestingly, CORM-3 did not influence the ZO- 1 and Occludin expression in HBMEC under inactivated neutrophils co-culture conditions. These results are consistent with our immunofluorescence staining (Figures 4D, E).

\section{DISCUSSION}

Although cell transplantation, drug and growth factor applications could reduce $\mathrm{BSCB}$ damage, the therapeutic efficacy also be affected as the restore of BSCB function (Kumar et al., 2016). Compare to the above treatments, carbon monoxide, as a gas molecule, could pass through the BSCB and reach the spinal segments, which are far from the primary damaged area.

As one of the degradation products of heme, CO has been proved with biological function including anti-inflammation, 

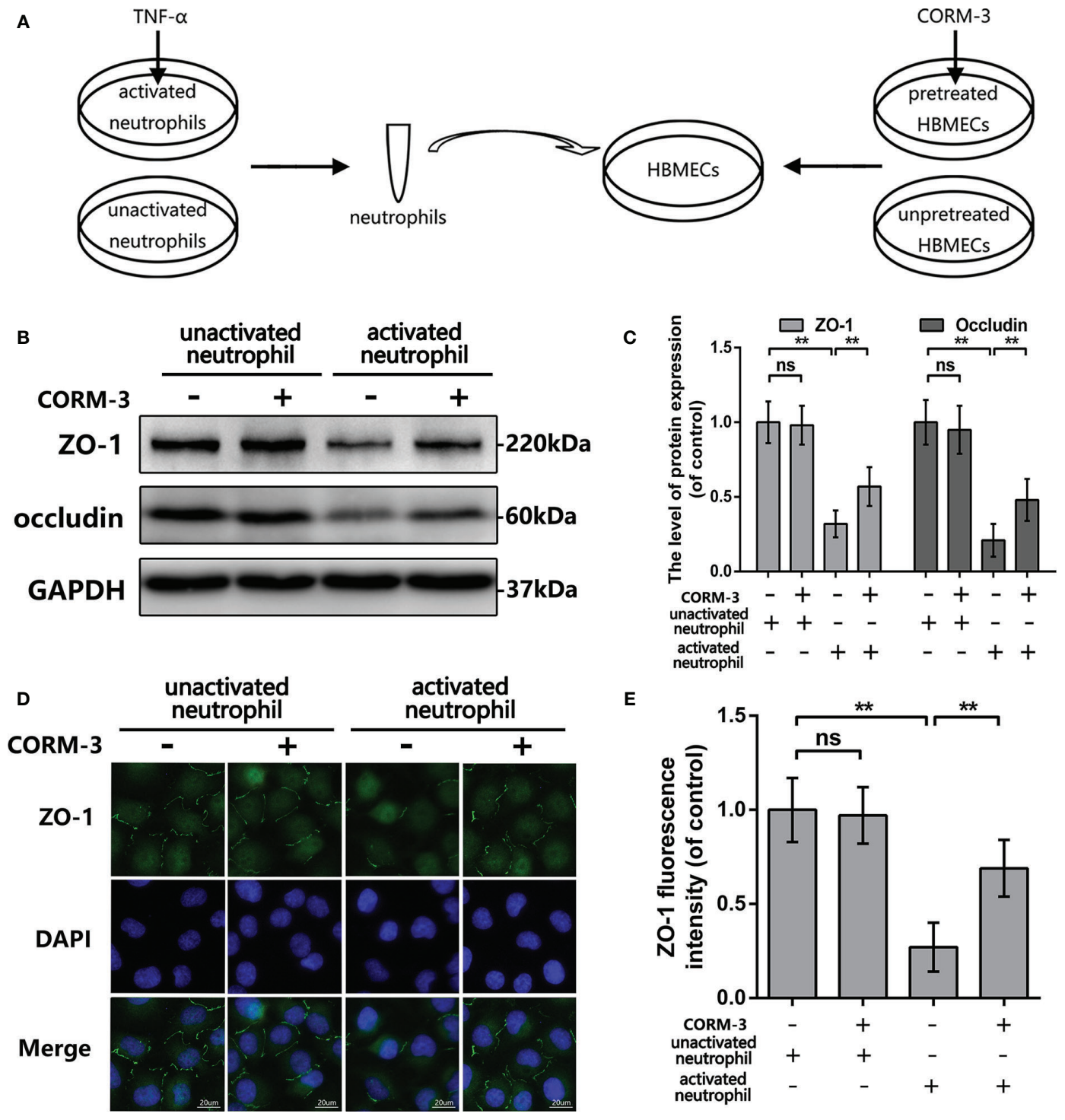

FIGURE 4 | CORM-3 alleviated TJ protein degradation induced by activated neutrophils in HMBECs, in vitro. (A) Schematic experimental settings for the neutrophils and HBMECs co-culture. (B, C) The protein expression of ZO-1 and Occludin in HBMECs treat as above. (D, E) Representative immunofluorescence staining and quantification data of ZO-1 in HBMEC. (bar: $20 \mu \mathrm{m}$ ). All data is denoted as mean \pm S.D. $(\mathrm{n}=5)$. ${ }^{* \star} P<0.01$. NS, No significant.

anti-apoptosis, and anti-oxidative at low dose (Motterlini and Otterbein, 2010; Choi et al., 2016). Several animal experiments proved that providing $\mathrm{CO}$ through inhaling or exogenic $\mathrm{CO}$ donors, the CORM-3, alleviates neural inflammation, neuron death and promotes neurogenesis (Zeynalov and Dore, 2009; Choi et al., 2016; Wang et al., 2018). However, to our knowledge, the effect of CO in BSCB protection after SCI is still unknown.

Following SCI, local mechanical impact to the spinal cord directly results in the vascular rupture and tissue destruction and consequently enhances the heme synthesis (extracted from hemoglobin/dying cells) (Gozzelino et al., 2010; GrochotPrzeczek et al., 2012). Simultaneously, HO-1was raised and activated compare to the uninjured spinal cord (Queiroga et al., 2015). These underlining mechanisms explains the SCIinitiated $\mathrm{CO}$ content variations in the spinal cord.

Normally, the BSCB blocks the entry of toxic substances into the spinal cord microenvironment. Succeeding acute spinal cord trauma, the BSCB disruption is defined as the fatal event which contributes to the secondary-injury and characterizes with the time dependence that initiates within $5 \mathrm{~min}$, peaks at $24 \mathrm{~h}$, and lasts for up to 28 days or longer and extends to the local domain of the spinal cord (Noble and Wrathall, 1989; Runge et al., 1997). Due to the barrier opening and chemokines secretion, the neutrophils infiltration further exacerbates BSCB damage by producing various matrix metallopeptidases (MMPs), especially MMP-9. And, the overproduction of MMP-9 facilitates the 
degeneration of TJ protein and basal lamina. In the mouse model of ischemic stroke, the CORM-3 administration by retro-orbital injection alleviated the $\mathrm{BBB}$ disruption and neurologic deficit in rat stroke model (Wang et al., 2018). However, whether CO protect BSCB following SCI remains to be studied. Our results demonstrated the BSCB permeation, neutrophils infiltration, TJ protein degeneration, and MMP-9 activity in spinal cord are increased after injury, but $\mathrm{CO}$ treatment reversed these phenomena. Previous studies reported carbon monoxide could regulate the ICAM expression, which is an adhesion molecule that is constitutively expressed in ECs at low levels but exhibits increased levels of expression under hypoxic conditions (Hopkins et al., 2004). Lee et al. reported that CORM-2 attenuates ICAM-1 expression in $P$. aeruginosa-induced lung inflammation mice and human pulmonary alveolar epithelial cells (Yeh et al., 2014). Adhesion molecules generally encoded by the transcription factor such as NF- $\kappa B$. In the TNF- $\alpha$ and IL- $1 \beta$ exposed human gingival fibroblasts, CORM-3 inhibited adhesion molecules expression by suppressing the NF- $\kappa \mathrm{B}$ pathway (Song et al., 2011). Additionally, Cunha and his colleagues demonstrated that the CO donor, dimanganese decacarbonyl, sustained the neutrophils adhesion and migration and ICAM-1 expression depending on soluble guanylate cyclase (sGC) activation (Lee et al., 2018). By the isolating the neutrophils from the PKG-deficient mice, they found the $\mathrm{HO} / \mathrm{CO} / \mathrm{PKG}$ pathway involves in neutrophil migration which might be further explain by the PKG effect on the NF- $\kappa B$ phosphorylation. However, Stirling et al. reported that inhibiting neutrophils by anti-Ly6G/Gr-1 aggravated the neurological outcome, which seems to be contrary to our results. This discrepancy results may be caused by the number of remaining neutrophils (Stirling et al., 2009). The use of an anti-Ly6G/Gr-1 antibody could abolish subtotal systemic neutrophils whereas CORM-3 acts by decreasing the neutrophils at the site of SCI.

Compare to wild-type mice, MMP-9 knockout mice exhibited the more BSCB integrity, the alleviation of neuroinflammation and significant locomotor recovery (Noble et al., 2002). Wang and his colleagues found that the MMP-9 expression and its colocalization level with pericytes were declined by the CORM-3 in the mouse stroke model (Wang et al., 2018). Similarly, our western blot and gelatin zymography results demonstrated that MMP-9 expressions and activities decreased after CORM-3 treatment in vivo and in vitro. Various studies investigated the NF- $\kappa \mathrm{B}$ could bind to the MMP-9 corresponding sequence and control its transcription (Mi et al., 2018; Muscella et al., 2018). In our in vitro researches, CORM3 sustained TNF- $\alpha$ stimulated the p65 nuclear transcription in neutrophils. But the carbon monoxide-induced the NF- $\kappa B$ regulation still needs to further explore. TNF- $\alpha$ activates I $\mathrm{B}$ kinase (IKK) and degrades I $\mathrm{B} B \alpha$, resulting in the liberation and translocation of $\mathrm{p} 65$, which then increases transcription of downstream mediators such as MMP-9 and ICAM-1. However, Wung and his colleagues showed that applying CO donors in ECs blocked the TNF- $\alpha$-induced p65 nuclear translocation by intervening its glutathionylation, but not the $\mathrm{I} \kappa \mathrm{B} \alpha$ degradation (Yeh et al., 2014). And the CO donors-caused intracellular ROS level slight increase and Nrf2 nuclear accumulation play the important roles in p65 glutathionylation. In brief, the low-level
ROS enhances the oxidation to reduction ratio of glutathione (GSSG/GSH) and Nrf2 activation mediated the expression of $\gamma$ glutamylcysteine synthetase, which is the rate-limiting enzyme in GSH synthesis (Yeh et al., 2014). These events medicated by CO administration contribute to proteins glutathionylation. The mechanism about CO-induced p65 inactivation in TNF- $\alpha$ exposed neutrophil requires investigation in detail.

Due to CO could competitively bind to hemoglobin with oxygen, it is considered as a toxic gas (Motterlini and Otterbein, 2010). Although CO has multiple biological activities at low concentrations, its therapeutic application is hampered by the lack of a safe and effective delivery (Queiroga et al., 2015). Continuous $\mathrm{CO}$ inhalation could rapidly elevate carboxyhemoglobin $(\mathrm{COHb})$ to toxic levels (> 15\%) (Otterbein et al., 2000). In our study, we did not detect $\mathrm{COHb}$ level in CORM-3 treated rats, but Prabhu et al. reported that the $\mathrm{COHb}$ level was stably maintained at $6 \%$ during the 24 days medication period in the mice administrating the CORM-3 (40 mg/kg/d) by intraperitoneal injection (Chen, 2014).

\section{DATA AVAILABILITY STATEMENT}

The datasets generated for this study are available on request to the corresponding authors.

\section{ETHICS STATEMENT}

The animal study was reviewed and approved by Animal Care and Use Committee of Wenzhou Medical University.

\section{AUTHOR CONTRIBUTIONS}

GZ wrote the paper, performed the experiments, and generated data. GZ, FZ, and ZL performed the experiments and generated data. YW, NT, and DZ analyzed data. GX, CX, and YZ contributed reagents and materials tools. $\mathrm{HX}$ and $\mathrm{XZ}$ conceived and designed the experiments. TZ, SW, YSW designed the experiments and helped write the manuscript. $\mathrm{HM}$ and WN revised article and provided data.

\section{ACKNOWLEDGMENTS}

This project was funded by the Wenzhou Science and Technology Bureau Foundation (Y20170083, Y20170092); National Natural Science Foundation of China (81601963, 81873992, 81572227); Zhejiang Provincial Natural Science Foundation of China (LY17H060009).

\section{SUPPLEMENTARY MATERIAL}

The Supplementary Material for this article can be found online at: https://www.frontiersin.org/articles/10.3389/fphar. 2020.00761/full\#supplementary-material

FIGURE S1 | CORM-3 inhibits NF- $\kappa B$ signaling activity after SCl. (A, B) The protein expression of $\mathrm{p}-\mathrm{p} 65$ and $\mathrm{p} 65$ in spinal cord at 3 days post-SCl. All data is denoted as mean \pm S.D. $(n=5) .{ }^{\star \star} P<0.01$. 


\section{REFERENCES}

Bartanusz, V., Jezova, D., Alajajian, B., and Digicaylioglu, M. (2011). The bloodspinal cord barrier: morphology and clinical implications. Ann. Neurol. 70, 194-206. doi: 10.1002/ana.22421

Chen, J. (2014). Heme oxygenase in neuroprotection: from mechanisms to therapeutic implications. Rev. Neurosci. 25, 269-280. doi: 10.1515/revneuro2013-0046

Choi, Y. K., Maki, T., Mandeville, E. T., Koh, S. H., Hayakawa, K., Arai, K., et al. (2016). Dual effects of carbon monoxide on pericytes and neurogenesis in traumatic brain injury. Nat. Med. 22, 1335-1341. doi: 10.1038/nm.4188

de Castro, R. C.Jr., Burns, C. L., McAdoo, D. J., and Romanic, A. M. (2000). Metalloproteinase increases in the injured rat spinal cord. Neuroreport 11, 3551-3554. doi: 10.1097/00001756-200011090-00029

Dilshara, M. G., Kang, C. H., Choi, Y. H., and Kim, G. Y. (2015). Mangiferin inhibits tumor necrosis factor- $\alpha$-induced matrix metalloproteinase- 9 expression and cellular invasion by suppressing nuclear factor- $\mathrm{\kappa B}$ activity. Bmb Rep. 48, 559-564. doi: 10.5483/BMBRep.2015.48.10.003

Fassbender, J. M., Whittemore, S. R., and Hagg, T. (2011). Targeting Microvasculature for Neuroprotection after SCI. Neurother. J. Am. Soc. Exp. Neurother. 8, 240-251. doi: 10.1038/jcbfm.2011.136

Giszter, S. F. (2008). Spinal cord injury: present and future therapeutic devices and prostheses. Neurotherapeutics 5, 147-162. doi: 10.1016/j.nurt.2007.10.062

Gozzelino, R., Jeney, V., and Soares, M. P. (2010). Mechanisms of cell protection by heme oxygenase-1. Annu. Rev. Pharmacol. Toxicol. 50, 323-354. doi: 10.1146/annurev.pharmtox.010909.105600

Grochot-Przeczek, A., Dulak, J., and Jozkowicz, A. (2012). Haem oxygenase-1: non-canonical roles in physiology and pathology. Clin. Sci. 122, 93-103. doi: 10.1042/CS20110147

Hopkins, A. M., Baird, A. W., and Nusrat, A. (2004). ICAM-1: targeted docking for exogenous as well as endogenous ligands. Adv. Drug Delivery Rev. 56, 763778. doi: 10.1016/j.addr.2003.10.043

Kim, K. M., Pae, H. O., Zheng, M., Park, R., Kim, Y. M., and Chung, H. T. (2007). Carbon monoxide induces heme oxygenase- 1 via activation of protein kinase R-like endoplasmic reticulum kinase and inhibits endothelial cell apoptosis triggered by endoplasmic reticulum stress. Circ. Res. 101, 919-927. doi: 10.1161/CIRCRESAHA.107.154781

Kumar, H., Ropper, A. E., Lee, S. H., and Han, I. (2016). Propitious Therapeutic Modulators to Prevent Blood-Spinal Cord Barrier Disruption in Spinal Cord Injury. Mol. Neurobiol. 54, 1-13. doi: 10.1007/s12035-016-9910-6

Kumar, H., Jo, M. J., Choi, H., Muttigi, M. S., Shon, S., Kim, B. J., et al. (2017). Matrix Metalloproteinase-8 Inhibition Prevents Disruption of Blood-Spinal Cord Barrier and Attenuates Inflammation in Rat Model of Spinal Cord Injury. Mol. Neurobiol. 55 (2018), 2577-2590. doi: 10.1007/s12035-017-0509-3

Lee, J. Y., Kim, H. S., Choi, H. Y., Oh, T. H., and Yune, T. Y. (2012). Fluoxetine inhibits matrix metalloprotease activation and prevents disruption of bloodspinal cord barrier after spinal cord injury. Brain A J. Neurol. 135, 2375. doi: 10.1093/brain/aws171

Lee, J. Y., Choi, H. Y., Ahn, H. J., Ju, B. G., and Yune, T. Y. (2014). Matrix metalloproteinase-3 promotes early blood-spinal cord barrier disruption and hemorrhage and impairs long-term neurological recovery after spinal cord injury. Am. J. Pathol. 184, 2985-3000. doi: 10.1016/j.ajpath.2014.07.016

Lee, C. W., Wu, C. H., Chiang, Y. C., Chen, Y. L., Chang, K. T., Chuang, C. C., et al. (2018). Carbon monoxide releasing molecule-2 attenuates Pseudomonas aeruginosa-induced ROS-dependent ICAM- 1 expression in human pulmonary alveolar epithelial cells. Redox Biol. 18, 93-103. doi: 10.1016/ j.redox.2018.07.001

Lin, W. P., Xiong, G. P., Lin, Q., Chen, X. W., Zhang, L. Q., Shi, J. X., et al. (2016). Heme oxygenase-1 promotes neuron survival through down-regulation of neuronal NLRP1 expression after spinal cord injury. J. Neuroinflamm. 13, 52. doi: 10.1186/s12974-016-0521-y

Lin, W., Wang, S., Yang, Z., Lin, J., Ke, Q., Lan, W., et al. (2017). Heme Oxygenase1 Inhibits Neuronal Apoptosis in Spinal Cord Injury through DownRegulation of Cdc42-MLK3-MKK7-JNK3 Axis. J. Neurotrauma 34, 695-706. doi: 10.1089/neu.2016.4608

Lu, T., Wu, X., Wei, N., Liu, X., Zhou, Y., Shang, C., et al. (2018). Lipoxin A4 protects against spinal cord injury via regulating Akt/nuclear factor (erythroid- derived 2)-like 2/heme oxygenase-1 signaling. Retour. Au Numéro 97, 905. doi: 10.1016/j.biopha.2017.10.092

Mi, B., Wang, J., Liu, Y., Liu, J., Hu, L., Panayi, A. C., et al. (2018). Icariin Activates Autophagy via Down-Regulation of the NF- KB Signaling-Mediated Apoptosis in Chondrocytes. Front. Pharmacol. 09, 605. doi: 10.3389/fphar.2018.00605

Motterlini, R., and Otterbein, L. E. (2010). The therapeutic potential of carbon monoxide. Nat. Rev. Drug Discovery 9, 728-743. doi: 10.1038/nrd3228

Muscella, A., Cossa, L. G., Vetrugno, C., Antonaci, G., and Marsigliante, S. (2018). Adenosine diphosphate regulates MMP2 and MMP9 activity in malignant mesothelioma cells. Ann. N. Y. Acad. Sci. 1431 (2018), 72-84. doi: 10.1111/ nyas. 13922

Neirinckx, V., Coste, C., Franzen, R., Gothot, A., Rogister, B., and Wislet, S. (2014). Neutrophil contribution to spinal cord injury and repair. J. Neuroinflamm. 11, 150. doi: 10.1186/s12974-014-0150-2

Noble, L. J., and Wrathall, J. R. (1989). Distribution and time course of protein extravasation in the rat spinal cord after contusive injury. Brain Res. 482, 5766. doi: 10.1016/0006-8993(89)90542-8

Noble, L. J., Donovan, F., Igarashi, T., Goussev, S., and Werb, Z. (2002). Matrix Metalloproteinases Limit Functional Recovery after Spinal Cord Injury by Modulation of Early Vascular Events. J. Neurosci. 22, 7526-7535. doi: 10.1523/ JNEUROSCI.22-17-07526.2002

Obermeier, B., Daneman, R., and Ransohoff, R. M. (2013). Development, maintenance and disruption of the blood-brain barrier. Nat. Med. 19, 15841596. doi: $10.1038 / \mathrm{nm} .3407$

Otterbein, L. E., Bach, F. H., Alam, J., Soares, M., Tao, L. H., Wysk, M., et al. (2000). Carbon monoxide has anti-inflammatory effects involving the mitogen-activated protein kinase pathway. Nat. Med. 6, 422-428. doi: 10.1038/74680

Palmer, A. M. (2010). The role of the blood-CNS barrier in CNS disorders and their treatment. Neurobiol. Dis. 37, 3-12. doi: 10.1016/j.nbd.2009.07.029

Pashevin, D. O., Nagibin, V. S., Tumanovska, L. V., Moibenko, A. A., and Dosenko, V. E. (2015). Proteasome Inhibition Diminishes the Formation of Neutrophil Extracellular Traps and Prevents the Death of Cardiomyocytes in Coculture with Activated Neutrophils during Anoxia-Reoxygenation. Pathobiology 82, 290-298. doi: 10.1159/000440982

Patterson, E. K., Fraser, D. D., Capretta, A., Potter, R. F., and Cepinskas, G. (2014). Carbon monoxide-releasing molecule 3 inhibits myeloperoxidase (MPO) and protects against MPO-induced vascular endothelial cell activation/dysfunction. Free Radic. Biol. Med. 70, 167-173. doi: 10.1016/j.freeradbiomed.2014.02.020

Penas, C., Verdú, E., Forés, J., Navarro, X., and Casas, C. (2010). Spinal cord injury induces endoplasmic reticulum stress with different cell-type dependent response. J. Neurochem. 102, 1242-1255. doi: 10.1111/j.1471-4159.2007.04671.x

Queiroga, C. S., Vercelli, A., and Vieira, H. L. (2015). Carbon monoxide and the CNS: challenges and achievements. Br. J. Pharmacol. 172, 1533. doi: 10.1111/ bph.12729

Runge, V. M., Wells, J. W., Baldwin, S. A., Scheff, S. W., and Blades, D. A. (1997). Evaluation of the temporal evolution of acute spinal cord injury. Invest. Radiol. 32, 105-110. doi: 10.1097/00004424-199702000-00006

Sharma, H. S. (2011). Early microvascular reactions and blood-spinal cord barrier disruption are instrumental in pathophysiology of spinal cord injury and repair: novel therapeutic strategies including nanowired drug delivery to enhance neuroprotection. J. Neural Transm. 118, 155-176. doi: 10.1007/ s00702-010-0514-4

Song, H., Zhao, H., Qu, Y., Sun, Q., Zhang, F., Du, Z., et al. (2011). Carbon monoxide releasing molecule- 3 inhibits concurrent tumor necrosis factor- $\alpha-$ and interleukin-1 $\beta$-induced expression of adhesion molecules on human gingival fibroblasts. J. Periodontal. Res. 46, 48-57. doi: 10.1111/j.16000765.2010.01307.x

Stirling, D. P., Liu, S., Kubes, P., and Yong, V. W. (2009). Depletion of Ly6G/Gr-1 leukocytes after spinal cord injury in mice alters wound healing and worsens neurological outcome. J. Neurosci. 29, 753-764. doi: 10.1523/ JNEUROSCI.4918-08.2009

Tahanian, E., Sanchez, L. A., Shiao, T. C., Roy, R., and Annabi, B. (2011). Flavonoids targeting of IkappaB phosphorylation abrogates carcinogeninduced MMP-9 and COX-2 expression in human brain endothelial cells. Drug Des. Devel. Ther. 5, 299-309. doi: 10.2147/DDDT.S19931

Wang, G., Hamid, T., Keith, R. J., Zhou, G., Partridge, C. R., Xiang, X., et al. (2010). Cardioprotective and antiapoptotic effects of heme oxygenase-1 in the failing 
heart. Circulation 121, 1912-1925. doi: 10.1161/CIRCULATIONAHA. 109.905471

Wang, L., Yao, Y., He, R., Meng, Y., Li, N., Zhang, D., et al. (2017). Methane ameliorates spinal cord ischemia-reperfusion injury in rats: Antioxidant, anti-inflammatory and anti-apoptotic activity mediated by $\mathrm{Nrf2}$ activation. Free Radic. Biol. Med. 103, 69-86. doi: 10.1016/j.freerad biomed.2016.12.014

Wang, J., Di, Z., Fu, X., Yu, L., Lu, Z., Gao, Y., et al. (2018). Carbon monoxidereleasing molecule-3 protects against ischemic stroke by suppressing neuroinflammation and alleviating blood-brain barrier disruption. J. Neuroinflamm. 15, 188. doi: 10.1186/s12974-018-1226-1

Wei, W., Shurui, C., Zipeng, Z., Hongliang, D., Hongyu, W., Yuanlong, L., et al. (2018). Aspirin suppresses neuronal apoptosis, reduces tissue inflammation, and restrains astrocyte activation by activating the Nrf2/HO- 1 signaling pathway. Neuroreport. 29 (2018), 524-531. doi: 10.1097/WNR.000000 0000000969

Wells, J. E., Rice, T. K., Nuttall, R. K., Edwards, D. R., Zekki, H., Rivest, S., et al. (2003). An adverse role for matrix metalloproteinase 12 after spinal cord injury in mice. J. Neurosci. 23, 10107-10115. doi: 10.1523/JNEUROSCI.23-3110107.2003

Yeh, P. Y., Li, C. Y., Hsieh, C. W., Yang, Y. C., Yang, P. M., and Wung, B. S. (2014). CO-releasing molecules and increased heme oxygenase-1 induce protein S-

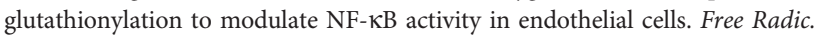
Biol. Med. 70, 1-13. doi: 10.1016/j.freeradbiomed.2014.01.042

Yu, F., Kamada, H., Niizuma, K., Endo, H., and Chan, P. H. (2008). Induction of mmp-9 expression and endothelial injury by oxidative stress after spinal cord injury. J. Neurotrauma 25, 184-195. doi: 10.1089/neu.2007.0438
Zeynalov, E., and Doré, S. (2009). Low Doses of Carbon Monoxide Protect Against Experimental Focal Brain Ischemia. Neurotox. Res. 15, 133. doi: 10.1007/ s12640-009-9014-4

Zhang, W., Tao, A., Lan, T., Cepinskas, G., Kao, R., Martin, C. M., et al. (2017). Carbon monoxide releasing molecule-3 improves myocardial function in mice with sepsis by inhibiting NLRP3 inflammasome activation in cardiac fibroblasts. Basic Res. Cardiol. 112, 16. doi: 10.1007/s00395-017-0603-8

Zhang, D., Tang, Q., Zheng, G., Wang, C., Zhou, Y., Wu, Y., et al. (2017). Metformin ameliorates BSCB disruption by inhibiting neutrophil infiltration and MMP-9 expression but not direct TJ proteins expression regulation. J. Cell. Mol. Med. 21 (2017), 3322-3336. doi: 10.1111/jcmm.13235

Zheng, G., Zhan, Y., Wang, H., Luo, Z., Zheng, F., Zhou, Y., et al. (2019). Carbon monoxide releasing molecule-3 alleviates neuron death after spinal cord injury via inflammasome regulation. EBioMedicine 40, 643-654. doi: 10.1016/ j.ebiom.2018.12.059

Conflict of Interest: The authors declare that the research was conducted in the absence of any commercial or financial relationships that could be construed as a potential conflict of interest.

Copyright $\odot 2020$ Zheng, Zheng, Luo, Ma, Zheng, Xiang, Xu, Zhou, Wu, Tian, Wu, Zhang, Ni, Wang, Xu and Zhang. This is an open-access article distributed under the terms of the Creative Commons Attribution License (CC BY). The use, distribution or reproduction in other forums is permitted, provided the original author(s) and the copyright owner(s) are credited and that the original publication in this journal is cited, in accordance with accepted academic practice. No use, distribution or reproduction is permitted which does not comply with these terms. 